\title{
The Intricate Role of Cold Gas and Dust in Galaxy Evolution at Early Cosmic Epochs
}

\author{
Dominik A. Riechers ${ }^{1}$, Peter L. Capak ${ }^{2}$ and Christopher L. Carilli ${ }^{3}$ \\ ${ }^{1}$ Department of Astronomy, Cornell University, Space Sciences Building, \\ Ithaca, NY 14853, USA \\ email: riechers@cornell.edu \\ ${ }^{2}$ Spitzer Science Center, California Institute of Technology, MC 220-6, \\ 1200 East California Boulevard, Pasadena, CA 91125, USA \\ ${ }^{3}$ National Radio Astronomy Observatory, PO Box O, Socorro, NM 87801, USA
}

\begin{abstract}
Cold molecular and atomic gas plays a central role in our understanding of early galaxy formation and evolution. It represents the component of the interstellar medium (ISM) that stars form out of, and its mass, distribution, excitation, and dynamics provide crucial insight into the physical processes that support the ongoing star formation and stellar mass buildup. We here present results that demonstrate the capability of the Atacama Large (sub-)Millimeter Array (ALMA) to detect the cold ISM and dust in "normal" galaxies at redshifts $z=5-6$. We also show detailed studies of the ISM in massive, dust-obscured starburst galaxies out to $z>6$ with ALMA, the Combined Array for Research in Millimeter-wave Astronomy (CARMA), the Plateau de Bure Interferometer (PdBI), and the Karl G. Jansky Very Large Array (VLA). These observations place some of the most direct constraints on the dust-obscured fraction of the star formation history of the universe at $z>5$ to date, showing that "typical" galaxies at these epochs have low dust content, but also that highly-enriched, dusty starbursts already exist within the first billion years after the Big Bang.
\end{abstract}

Keywords. galaxies: evolution, galaxies: high-redshift, galaxies: ISM, cosmology: early universe, radio lines: galaxies, submillimeter

\section{Introduction}

Over the past two decades, great progress has been made in measuring the evolution of the volume density of the star formation rate (SFR) in galaxies through cosmic times ("star formation history of the universe"). Detailed studies have shown a steep decline in the SFR density since $\sim 3.5 \mathrm{Gyr}$ ago (corresponding to $z \sim 2$ ). Galaxies have formed 10-30 times more stars in the peak epoch of star formation activity at $z=1-3$, and half of the stellar mass that is seen in galaxies today was formed before $z=1.3$. Beyond the peak at $z \sim 2$, the SFR density appears to decline toward earlier epochs, approaching the present-day value likely at $z \gtrsim 7$ (see Madau \& Dickinson 2014 for a recent review). The higher SFRs in galaxies at high redshifts are likely largely driven by increased cold gas mass fractions, and thus, more available "fuel" for star formation in both "typical" and starburst galaxies (which reach SFRs that are at least an order of magnitude higher than the most extreme starbursts in the nearby universe), rather than greatly increased star formation efficiencies (see, e.g., Carilli \& Walter 2013 for a recent review).

A large fraction of the cosmic star formation activity is obscured by dust, with higher obscuration in more intensely star-forming galaxies - which typically contain more gas and dust. To measure the total SFR density, it thus is necessary to obtain measurements of both the direct stellar light from young, recently formed massive stars at ultraviolet wavelengths, and of the fraction of the stellar light absorbed and re-processed by dust, 


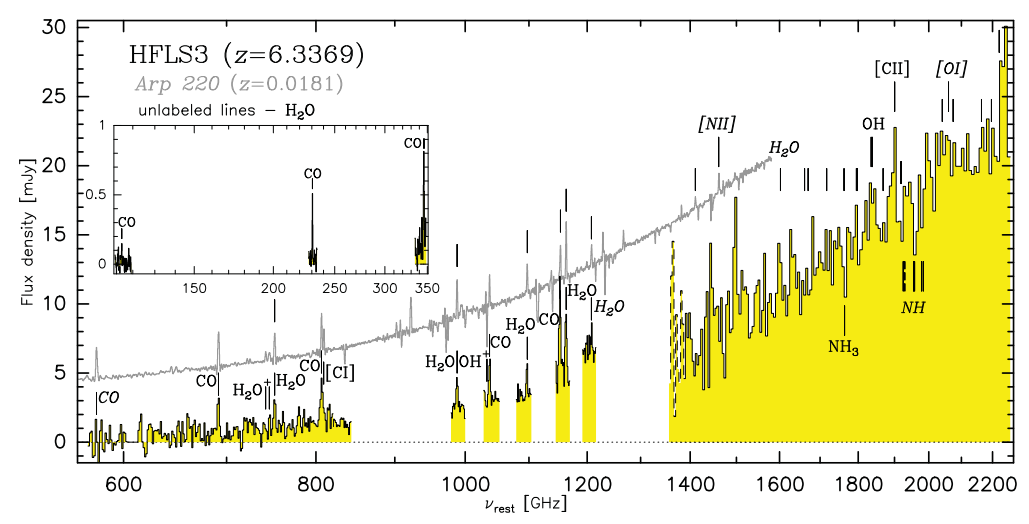

Figure 1. Wide-band (sub-)millimeter spectrum (histogram) of the $z=6.34$ massive dust-obscured starburst galaxy HFLS3, as observed with CARMA, the PdBI, the VLA (inset), and Z-spec mounted on the Caltech Submillimeter Observatory (CSO; Riechers et al. 2013). The gray line shows the Herschel/SPIRE spectrum of the nearby starburst Arp 220 for comparison.

which is re-emitted as thermal radiation at infrared wavelengths. Based on measurements with the Spitzer Space Telescope and the Herschel Space Observatory (e.g., Le Floc'h et al. 2005; Magnelli et al. 2013), the contribution of dust-obscured star formation to the SFR density is now fairly well understood out to $z=2-3$; however, at $z>3$, measurements are limited to only few, extremely luminous systems, rendering existing constraints at even higher redshifts $(z=5-6)$ difficult to generalize.

Some of the most extremely star-forming galaxies at high redshifts are virtually completely enshrouded in dust, such that the most distant among them may remain undetected even in the most sensitive studies at rest-frame ultraviolet/optical wavelengths (e.g., Daddi et al. 2009; Walter et al. 2012). These galaxies are detected in sensitive surveys at submillimeter (i.e., rest-frame far-infrared) wavelengths where the dust becomes optically thin and re-emits the re-processed stellar light. However, owed to the "negative $K$-correction" in the traditional (sub-)millimeter observing bands at $850 \mu \mathrm{m}$ and $1.2 \mathrm{~mm}$ (e.g., Blain et al. 2002), surveys at these wavelengths are only weakly sensitive to redshift at $z \gtrsim 2$, making it difficult to systematically identify the rare, most distant starbursts at the highest redshifts $(z=5-6)$ among the much more numerous dusty galaxies at lower $z$.

In the following, we describe some recent progress in the identification and detailed study of the most luminous, dust-obscured massive starbursts at $z>5$, and in obtaining some of the first direct constraints on the interstellar gas and dust content of "typical" galaxies at $z=5-6$. Much of this progress is driven by the recent availability of ALMA, as well as sensitive large-area, multi-color (sub-)millimeter surveys, in particular those with the Spectral and Photometric Imaging Receiver (SPIRE) instrument on board Herschel.

\section{Uncovering the Most Distant Dust-Obscured Starbursts}

With the advent of sensitive surveys at 250, 350, and $500 \mu \mathrm{m}$ wavelengths with $\mathrm{Her}$ schel/SPIRE, it has become possible to develop a new technique to measure approximate, color-based photometric redshifts for extremely dust-obscured galaxies where no traditional redshift measurements are available. SPIRE typically probes low- and intermediate-redshift galaxies on the Rayleigh-Jeans tail or across the peak of their dust spectral energy distributions (SEDs), making them appear "blue" or "green" in color-composite SPIRE maps. For dusty starbursts with typical dust temperatures of $T_{\text {dust }}=30-60 \mathrm{~K}$, the SPIRE bands probe rest-frame wavelengths shortward of the peak of 

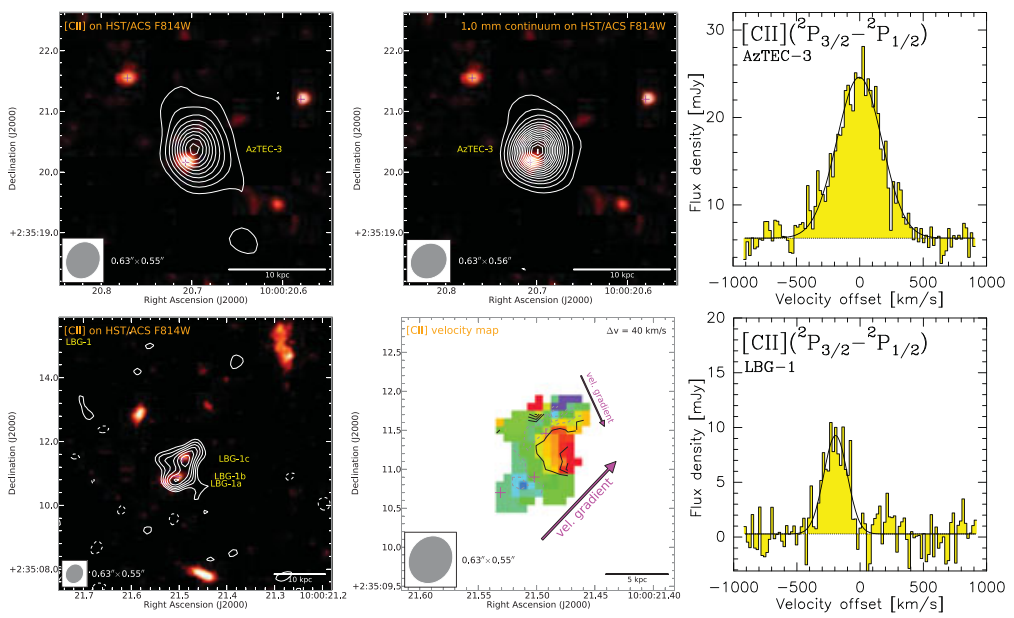

Figure 2. ALMA detections of [CII] and rest-frame $157.7 \mu \mathrm{m}$ continuum emission (contours) toward the $z=5.3$ dusty starburst AzTEC-3 (top panels), and the $z=5.3$ Lyman-break galaxy LBG-1 (bottom panels) on a Hubble Space Telescope Advanced Camera for Surveys (HST/ACS) image in the $\mathrm{F} 814 \mathrm{~W}$ filter (Riechers et al. 2014). The resolution (synthesized beam size) of the ALMA data is shown in the bottom left corner of the left and middle panels. Left: Line-averaged [CII] emission maps over 466 (top; AzTEC-3) and $330 \mathrm{~km} \mathrm{~s}^{-1}$ (bottom; LBG-1). Continuum emission has been subtracted from the AzTEC-3 map, and is negligible in comparison to the line strength in the case of LBG-1. Top middle: $157.7 \mu$ m continuum emission toward AzTEC-3. Bottom middle: [CII] velocity map (first moment) toward LBG-1. Right: [CII] line spectra (histograms), and Gaussian fits to the line profiles (curves). All velocities are relative to $z=5.2988$. their dust SEDs at $z \gtrsim 3.5$ - making them appear "red" (i.e., $S_{250 \mu \mathrm{m}}<S_{350 \mu \mathrm{m}}<S_{500 \mu \mathrm{m}}$ ), and the redder the more distant. We have verified this technique though detailed followup of HFLS3, the most promising, "ultra-red" candidate in the Herschel Multi-tiered Extragalactic Survey (HerMES) SPIRE data, yielding a redshift of $z=6.34$ based on molecular and atomic fine structure lines from the star-forming interstellar gas (Fig. 1; Riechers et al. 2013 - the highest redshift for a dust-obscured starburst previously measured was $z=5.30$; Riechers et al. 2010; Capak et al. 2011). A systematic search in the HerMES SPIRE data has revealed tens of additional "red" candidates (Dowell et al. 2014). Spectroscopic follow-up of 14 sources confirms that most (79\%) are at $z>4$, and $36 \%$ are even at $z>5$ (D. A. Riechers et al., in prep.; see also Clements et al., this volume).

\section{ISM and Dust-Obscured SFR in "Typical" $z=5-6$ Galaxies}

Using ALMA in cycle-0, we have observed the center of a galaxy proto-cluster at $z=5.3$ in the Cosmological Evolution Survey (COSMOS) field, targeting the [CII] ISM cooling line and rest-frame $157.7 \mu \mathrm{m}$ continuum emission. We have detected strong [CII] and continuum emission toward AzTEC-3, a gas-rich, dusty starburst in the center of the proto-cluster. Moreover, we have detected strong, dynamically-resolved [CII] emission toward LBG-1, a triplet of "typical" (i.e., $\sim L_{\mathrm{UV}}^{*}$ at $z=5.3$ ), Lyman-break galaxies at $\sim 95 \mathrm{kpc}$ distance from the dusty starburst - demonstrating that ALMA can detect and resolve the star-forming interstellar gas in "normal" galaxies back to the first billion years of cosmic time (Fig. 2; Riechers et al. 2014). We have then used ALMA in the following cycle to target 8 more "typical" $z=5-6$ galaxies (and one quasar) in the COSMOS field, and to obtain deeper observations of LBG-1. We detect [CII] emission in all nine "typical" galaxies and the dust continuum in four of them, also obtaining a statistical detection of the dust in a stack of the individually-undetected galaxies (Capak et al. 2015). Based on the rest-frame far-infrared detections of the dust, we find lower infrared luminosities than 

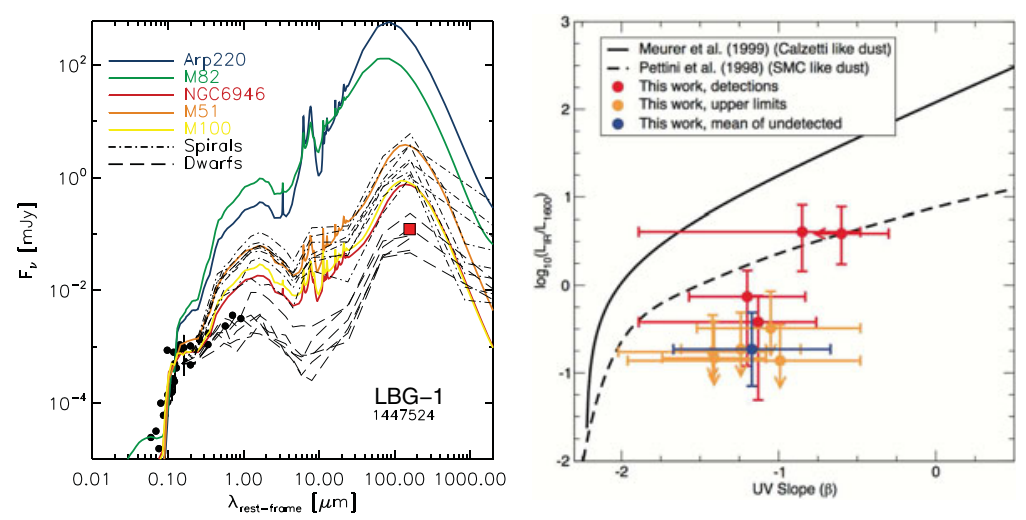

Figure 3. Left: Spectral energy distribution of the "typical" $z=5.3$ galaxy LBG-1 (data points), and nearby galaxy templates (lines; Riechers et al. 2014; including the data from Capak et al. 2015). The red square shows the ALMA measurement at rest-frame $157.7 \mu \mathrm{m}$. All templates are matched to the observed $i$-band flux of LBG-1. Right: Relation between the infrared excess (IRX, as expressed by the ratio between the rest-frame infrared and $1600 \AA$ ultraviolet luminosities) and the ultraviolet continuum slope $(\beta)$ for 9 "typical" galaxies at $z=5-6$ (and a stacked detection of the 5 upper limits), compared with models for near-solar metallicity (solid line; consistent with "typical" galaxies at $z<3$ ) and lower-metallicity (dashed line) dust (Capak et al. 2015).

expected from the dust properties of nearby spirals and starbursts. However, the dust properties of our "typical" $z=5-6$ galaxies are consistent with those of low-metallicity dwarf galaxies (Fig. 3, left). This finding agrees with their positions on the IRX- $\beta$ relation between the infrared excess and the ultraviolet continuum slope (a common measure for dust extinction), which are inconsistent with what is expected for standard, "Calzettilike" dust, but agree better with "SMC-like" dust, as found in lower-metallicity galaxies (Fig. 3, right). This suggests an evolution in the dust properties between $\sim L_{\mathrm{UV}}^{*}$ galaxies at $z<3$ and those at $z=5-6$, and that standard assumptions based on the ultraviolet continuum slope would overpredict the dust-obscured fraction of the SFR in our sample typically by factors of at least $\sim 12$. Taken at face value, our measurements would imply a downward correction of the total SFR density at $z=5-6$ by $\lesssim 40 \%$ (Capak et al. 2015).

Acknowledgments: It is a pleasure to thank our collaborators on the research projects discussed here, in particular F. Bertoldi, C. M. Bradford, C. M. Carollo, C. M. Casey, D. L. Clements, P. Cox, C. D. Dowell, A. Karim, I. Pérez-Fournon, O. Ilbert, R. J. Ivison, G. Jones, O. LeFevre, S. Lilly, E. Schinnerer, N. Z. Scoville, K. Sheth, V. Smolčić, M. Viero, L. Yan, M. Yun, and the COSMOS and HerMES collaborations.

\section{References}

Blain, A. W., Smail, I., Ivison, R. J., Kneib, J.-P., \& Frayer, D. T. 2002, PhR, 369, 111

Capak, P. L., Riechers, D., Scoville, N. Z., et al. 2011, Nature, 470, 233

Capak, P. L., Carilli, C., Jones, G., et al. 2015, Nature, 522, 455

Carilli, C. L. \& Walter, F. 2013, ARA\&A, 51, 105

Daddi, E., Dannerbauer, H., Krips, M., et al. 2009, ApJ, 695, L176

Dowell, C. D., Conley, A., Glenn, J., et al. 2014, ApJ, 780, 75

Le Floc'h, E., Papovich, C., Dole, H., et al. 2005, ApJ, 632, 169

Madau, P. \& Dickinson, M. 2014, ARA\& $A, 52,415$

Magnelli, B., Popesso, P., Berta, S., et al. 2013, A\& $\mathcal{E}$, 553, 132

Riechers, D. A., Capak, P. L., Carilli, C. L., et al. 2010, ApJ, 720, L131

Riechers, D. A., Bradford, C. M., Clements, D. L., et al. 2013, Nature, 496, 329

Riechers, D. A., Carilli, C. L., Capak, P. L., et al. 2014, ApJ, 796, 84

Walter, F., Decarli, R., Carilli, C., et al. 2012, Nature, 486, 233 